\title{
Meal Data Time Point Reference
}

National Cancer Institute

\section{Source}

National Cancer Institute. Meal Data Time Point Reference. NCI Thesaurus. Code

C162264.

The point in time that acts as a fixed reference point to meal data. 